\title{
Two Questions of Research on the Fairing for C- Bézier Curves
}

\author{
Z. N. Li \\ Department of Mathematics \\ Yinchuan Energy Institute \\ China
}

\author{
H. Zhou \\ Department of Mathematics \\ Yinchuan Energy Institute \\ China
}

\author{
Y. L. Chen \\ Department of Mathematics \\ Yinchuan Energy Institute \\ China
}

\begin{abstract}
This thesis talks about the preliminary study on the modification of the new C-Bézier curves, Asuccinct for mulafor calculating the derivative of the degree $3 \mathrm{C}$-Bezier curve is given. It studies the four order C-Bézier curve fairing problem. Fairing C-Bézier curves are satisfied by adjusting the shape of the parameter $\alpha$ on the basis of applying energy.
\end{abstract}

\section{Keywords-CAGD; C-Bézier curves; fairing, stitching}

\section{INTRODUCTION}

The term CAGD (Computer Aided Geometric Design)was proposed in 1974 at an international conference on the American Jewish University by Barnhill and Riesenfeld [1], in order to describe the computer-aided design in mathematical aspects. It is explained by means of theoretical and mathematical methods and computer technology to solve integration computer-aided design in the various mathematical problems. A mathematical model, making the design both for computer processing, can effectively meet the geometrical shape description and requirements, and makes it easy to shape the transmission of information and exchange product data, It is the theoretical foundation and key technologies in CAD.

In this section we describe the overview development of the curve and surface fairing research. The smooth curves and surfaces researching began in the early 1960s, which open up the first domestic research direction in 1974 by Professor Su Buqin. So many fairing methods have been proposed through the efforts of domestic and foreign scholars: on B-spline curve fairing of method by "node removal and insertion”(Farin[2]); using parametric cubic spline curve and local fairing method bicubic spline surfaces (Kjellander);Spacing for three parameters spline curve fairing and fairing energy grid method (Hosaka [3]);B-spline surface fairing energy method (Lott[4]); Spline-based method [5]; fairing curved method of grid lines [6]. These methods generally fall into two categories:

1)The fairing method, such as energy method, has transformed the fairing issue which contains data points bias and fairing the weighted average of the two parts of the objective function into minimization problems. Fairing is to use all the data points to modify.

2)Local fairing method, such as spline-based method and the node of insertion and deletion method, is the choice of site modification method. It is based on the assumption that most of the data points are good and modifies the few bad points singled out individually. However, when consecutive multiple dead pixels, it is often difficult to deal with. These methods are more classic. It is not difficult to find some connection between several methods in essence. It is worth mentioning that in 2002 Cai ZhongYi [7] proposed fairing finite element method, which has opened up new avenues of research. The method uses Hermite unit minimization objective function and reproduction of $C^{1}, C^{2}$ surface audience of continuous. This calculation method combined with the finite element interpolation fairing suppressed energy input data noise impact. High precision surface reconstruction fairing is good. Gan Yi [8], who has given a genetic algorithm-based surface fairing curve greatly improved the efficiency of computing. Using multi-resolution algorithms as well as the small waves emerged in recent years [9] of high efficiency, fairing can be done in linear time. However, in the fairing data compression, it can not handle the boundary constraints. Energy method is still a widely used method of curve and surface fairing can handle boundary constraints. It is suitable for large deflection and closed curves and surfaces smooth among the many methods fairing.

The unit normal vector which equidistant curves and surfaces (offset) in the formula include square root term and it is not an accurate representation of its equidistant curve (surface), which can not be treated with common systems using the same form CAGD curve (surface), so we have to use various means to offset approximating. In the equidistance line approximation algorithm, the literature [10] introduces a fairing and gives the fairing and approximation methods of the nonuniform rational B-spline curve which represents the fairing equidistance line. It combines approximate equidistance line with fairing in order to solve the bizarre case in part. In reverse engineering surface modeling based on digital things, fairing surface becomes particularly important, because the lack of the 
necessary feature information (refer continuity requirements information) and the presence of digital error.

Therefor, reverse engineering of CAD modeling for complex surface profile of the product, with further studies of fairing surface fitting technology items, making surface fitting not only has high accuracy, but also has good concerned fairing.

\section{DEFINITION AND PROPERTIES OF C-BÉZIER CURVES}

Give the definition and properties of C-Bézier curve.

Definition 2. 1 Set $\vec{q}_{0}, \vec{q}_{1}, \vec{q}_{2}, \vec{q}_{3} \in \mathfrak{R}^{3}$ or $\mathfrak{R}^{2}$ is four control point, $\alpha$ is arbitrary real number, and $0<\alpha \leq \pi$, so the curve

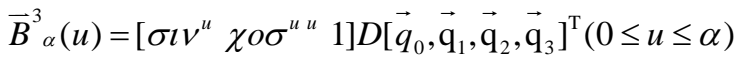

Where

$$
\begin{gathered}
D=\frac{1}{\alpha-s}\left[\begin{array}{cccc}
c & 1-c-m & m & -1 \\
-s & (\alpha-k) m & -m & 0 \\
-1 & m & -m & 1 \\
\alpha & -(\alpha-k) m & k m & 0
\end{array}\right] \\
s=\sin \alpha, c=\cos \alpha, K=\frac{\alpha-s}{1-c}, m=\left\{\begin{array}{l}
1, \\
\frac{s}{\alpha-2 k}, \\
\alpha=\pi \\
0<\alpha \leq \pi
\end{array}\right.
\end{gathered}
$$

is called three C-Bézier curves . Formula (1) can be represented as:

$$
\vec{B}_{\alpha}^{3}(u)=Z_{0}(u) \vec{q}_{0} Z_{1}(u) \vec{q}_{1_{+}} Z_{2}(u) \vec{q}_{2+} Z_{3}(u) \vec{q}_{3}
$$

where

$$
Z_{0}(u)=\frac{(\alpha-u)-\sin (\alpha-u)}{\alpha-\sin \alpha}
$$

$$
\begin{gathered}
Z_{3}(u)=\frac{u-\sin u}{\alpha-\sin \alpha}, \\
Z_{1}(u)=m\left[\frac{1-\cos (\alpha-u)}{1-\cos \alpha}-Z_{\mathrm{o}}(u)\right], \\
Z_{2}(u)=m\left[\frac{1-\cos u}{1-\cos \alpha}-Z_{3}(u)\right]
\end{gathered}
$$

is called basis functions of three C-Bézier curve . They meet the rights of non-negative, where (1) $\sum_{i=0}^{3} Z_{i}(u) \equiv 1^{(2)} Z_{i}(u) \geq 0(i=0,1,2,3)$

There are many similar basis functions between Bézier curve and C-Bézier curve and have three similar right with non-negative.

Properties of the curve [11]: like interpolation of endpoint, the convex hull, discrete properties can also be an accurate representation of a quadratic curve, which depends on $\alpha$ parameter increasing the degree of freedom of the curve constructed. The following properties of a limit is given.

Lemma 2. 1 [12] The limit of Second C-Bézier curve is a quadratic Bézier curve. so does three C-Bézier curve.

Theorem 2. 1 The derivative vector of the three C-Bézier curve (1) is

$$
\begin{aligned}
& \qquad \frac{d \vec{B}_{\alpha}^{3}(u)}{d(u)}=\frac{1}{\alpha}\left[\begin{array}{lll}
\sin u \quad \cos u & 1
\end{array}\right] M M^{*}\left[\begin{array}{lll}
\vec{a}_{1} & \vec{a}_{2} & \vec{a}_{3}
\end{array}\right]^{T} \\
& \text { where } \vec{a}_{1}=\vec{q}_{1}-\vec{q}_{0} ; \vec{a}_{2}=\vec{q}_{2}-\vec{q}_{1} ; \vec{a}_{3}=\vec{q}_{3}-\vec{q}_{2}
\end{aligned}
$$

$$
M^{*}=\operatorname{diag}\left[1, \frac{\alpha-\sin \alpha}{2 \sin \alpha-\alpha-\alpha \cos \alpha}, 1\right]
$$

$\frac{d \vec{B}_{\pi}^{3}(u)}{d(u)}=\frac{1}{\pi}\left[\begin{array}{lll}\sin u & \cos u & 1\end{array}\right]\left[\begin{array}{ccc}0 & 1 & 0 \\ 1 & 0 & -1 \\ 1 & 0 & 1\end{array}\right]\left[\begin{array}{lll}\vec{a}_{1} & \vec{a}_{2} & \vec{a}_{3}\end{array}\right]^{T}$

where $\vec{a}_{2}=\vec{q}_{2}-\frac{\pi}{2} \vec{q}_{1}$. This shows that the characteristic of C-Bézier curves is not established with Bézier curve.

\section{THE CURVE FAIRING APPROXIMATION OF C-BÉZIER}

In this section we study curve fairing approximation of CBézier. Literature [13] studies C-Bézier curve shape modification method, and it is proposed to adjust the interpolation of a point of control C-Bézier curve method. It also shows the effect of moving control points on the curve.

Consider

Question 1: We know the data sequence of points $\left\{\vec{p}_{i}\right\}(i=0,1, \cdots, n) \in \mathfrak{R}^{2}$,

Seeking a C-Bézier curves: $\Gamma: \vec{r}=\vec{B}_{\alpha}(t), t \in[0, \alpha]$, making the end of $p_{i} \in$ Tradius vector. The solution is as follows:

First, we will parameter values of data points, which can be standardized cumulative chord length parameterization,

Denoted as $: l_{i}=\left|\vec{p}_{i}-\vec{p}_{i-1}\right|, L=\sum_{i=0}^{n} l_{i},(i=0,1, \cdots, n)$, thus we have:

$$
\left\{\begin{array}{l}
t_{0}=0 \\
t_{1}=\alpha\left|\vec{p}_{1}-\vec{p}_{\mathrm{o}}\right| / L \\
\vdots \\
t_{n}=\alpha \sum_{i=0}^{n} t_{i} / L
\end{array}\right.
$$

So, nonlinear equations is the solution for normalization of the above problems: 


$$
\sum_{i=0}^{3} Z_{k}\left(t_{i}\right) \vec{q}_{k}=\vec{p}_{i}(i=0,1, \cdots, n) \quad(k=0,1, \cdots, n)
$$

If we have written in matrix form

$$
A Q=P
$$

Where $Q=\left[\vec{q}_{0}, \vec{q}_{1}, \vec{q}_{2}, \vec{q}_{3}\right]^{T}, \vec{q}_{0}, \vec{q}_{1}, \vec{q}_{2}$, $\vec{q}_{3} \in \mathfrak{R}^{3}$ or $\mathfrak{R}^{2}$ are four control points of the C-Bézier curve, $P=\left[\vec{p}_{0}, \vec{p}_{1}, \cdots, \vec{p}_{n}\right]^{T}$.

$$
A=\left[\begin{array}{cccc}
Z_{0}\left(t_{0}\right) & Z_{1}\left(t_{0}\right) & Z_{2}\left(t_{0}\right) & Z_{3}\left(t_{0}\right) \\
Z_{0}\left(t_{1}\right) & Z_{1}\left(t_{1}\right) & Z_{2}\left(t_{1}\right) & Z_{3}\left(t_{1}\right) \\
\cdots & \cdots & \cdots & \cdots \\
Z_{0}\left(t_{n}\right) & Z_{1}\left(t_{n}\right) & Z_{2}\left(t_{n}\right) & Z_{3}\left(t_{n}\right)
\end{array}\right]
$$

$Z_{k}(t) \quad(k=0,1, \cdots, n)$ is basis functions of C-Bézier curve.

There is no exact solution on (8)equations under normal circumstances, there is no strict interpolated curve through these data points, but we can find these data points of the least squares approximation solution. That parameter polynomial curve $\Gamma: \vec{r}=\vec{B}_{\alpha}(t), \sum_{i=0}^{n}\left|\vec{B}_{\alpha}\left(t_{i}\right)-\vec{p}_{i}\right|^{2}=\min$.

$A$ is the full column rank, that $\operatorname{rank}(A)=4$, so (8) orthogonal equations of corresponding Guass: $A^{T} A D=A^{T} P$ so:

$$
D=A^{+} P
$$

where : $A^{+}=\left(A^{T} A\right)^{-1} A^{T}$

Obtaining C-Bézier curve control points $\vec{q}_{0}, \vec{q}_{1}, \vec{q}_{2}, \vec{q}_{3}$ by Eq. (9) in order to get the solution of the problem 1.

Question 2: How to determine the parameters in order to correspond fairing of $\Gamma: \vec{r}=\vec{B}_{\alpha}(t)$ C-Bézier curve.

The shape of C-Bézier curve is related with $\alpha$ parameters. For fixed controling vertices, curve is moving away from the control polygon and tending to flatten with parameters of $\alpha$ gradual increases.

According to fairing criteria, applied energy method may establish fairing constraint equation

$$
E=\int_{0}^{\alpha}\left\|\vec{B}_{\alpha}(t)\right\|^{2} d t=\min
$$

Here we are seeking specific expression of $E$ fairing amount of fourth-order C-Bézier curve, By(7)it is

$$
{\overrightarrow{B^{\prime \prime}}}_{\alpha}(t)=\overrightarrow{T^{\prime \prime}} B D 0<\alpha \leq \pi, 0<t \leq \alpha
$$

Where, $\overrightarrow{T^{\prime \prime}}=[-\sin t,-\cos t, 0,0], \mathrm{D}$ and $\mathrm{B}$ are same with (10) formula, which has the

$$
E=(B D)^{T}\left[\begin{array}{cccc}
-\frac{1}{4} \sin ^{2} \alpha+\frac{1}{2} \alpha & \frac{1}{2} \sin ^{2} \alpha & 0 & 0 \\
\frac{1}{2} \sin ^{2} \alpha & \frac{1}{4} \sin ^{2} \alpha+\frac{1}{2} \alpha & 0 & 0 \\
0 & 0 & 0 & 0 \\
0 & 0 & 0 & 0
\end{array}\right](B D)
$$

We will consider deviation curve and minimum of fairing amount during the fairing, so integrating issues (1) and issues (2) we can let the energy of the whole system as $E \rightarrow$ min

So $\alpha$ parameters and control points can be obtained after fairing, but we hope we control curve points through the whole designing. Let $\vec{p}_{0}=\vec{q}_{0}, \vec{p}_{i}=\vec{q}_{3}$, we can get the $E$ parameters and the $\vec{q}_{1}, \vec{q}_{2}$ partial derivatives of control vertices, so as to achieve the purpose of the fairing.

From the variational principle it has

$$
\frac{\partial E}{\partial \alpha}=0 \frac{\partial E}{\partial \vec{q}_{1}}=0 \frac{\partial E}{\partial \vec{q}_{2}}=0
$$

Integrating issues (1) and issues (2) we give the C-Bézier curve fairing and approximation algorithm. It is as follows:

Step1:Method using cumulative chord length parameterization of data points

Step2: $\alpha, \vec{q}_{1}, \vec{q}_{2}$ determined by the (12) equation

Step3:Let the values of $\alpha, \vec{q}_{1}, \vec{q}_{2}$ into (8), thereby we can obtain the corresponding curve of fairing and approximation $\Gamma: \vec{r}=\vec{B}_{\alpha}(t) \in \mathfrak{R}^{2}, t \in[0, \alpha]$

Note: The fairing and approximation for higher-order CBézier curves and surfaces can be similarly treated. It is omitted here.

\section{CALCULATION EXAMPLE}

A set of data points $\vec{P}_{i}\left(x_{i}, y_{i}\right)(i=0,1, \cdots, 9)$ are given as the following table:

table i. A Set Of Data Points $\vec{P}_{i}\left(x_{i}, y_{i}\right)$

\begin{tabular}{|c|c|c|c|c|c|c|c|c|c|c|}
\hline $\boldsymbol{i}$ & 0 & 1 & 2 & 3 & 4 & 5 & 6 & 7 & 8 & 9 \\
\hline$x_{i}$ & 0. & 1 & 0. & 1.5 & 1.2 & 2. & 1. & 2 & 4.5 & 6 \\
\hline$y_{i}$ & 0. & 1 & 1 & 0.8 & 2.1 & 2. & 2. & 2 & 2 & 1 \\
\hline
\end{tabular}

Given a set values of $\alpha$, we will get a different energy, as shown in Table 2: 
TABLE II. THE ENERgy CHANGES OF the VALUE of $\alpha$ ARE SHOWN

\begin{tabular}{|c|c|c|c|c|c|c|}
\hline$\alpha$ & 0. & 0. & 1 & 1. & 2. & 2.6 \\
& 54 & 8 & & 137 & 0 & \\
\hline$E$ & 37. & 33. & 26. & 23. & 24. & 31. \\
& 55 & 27 & 26 & 28 & 52 & 219 \\
\hline
\end{tabular}

The fairing minimum energy is 23. 28 after fairing from the table when $\alpha=1.137$.

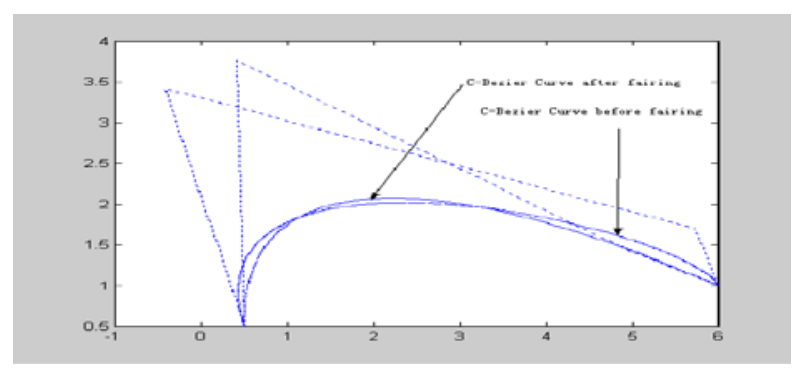

FIGURE 1: FAIRING AROUND THE C-BÉZIER CURVE

\section{CONCLUSIONS}

The nature and definition of C-Bézier curves are given firstly and carefully studied when $\alpha \rightarrow 0^{+}$, the Curve of $B\left(t ; \alpha_{1}\right)$ and $B\left(t ; \alpha_{2}\right)$ are intersecting ( $\alpha_{1} \neq \alpha_{2}$ ). On the basis of the energy law, making the curve is given by adjusting the minimum energy so as to achieve the purpose of fairing.

\section{ACKNOWLEDGEMENT}

This work was supported by Yinchuan Energy Institute Research: 2012-KY-P-35.

\section{REFERENCES}

[1] Piehl. L, Tiller. W. The NURBS Book[M]. 2nd ed. Berlin: Springer, 1997:201-230.

[2] Jianhua Fan, Jiwen Zhang, Yijie Wu. C-Bézier curve shape changes [J]. Journal of Software. 2002, 13(11):2194-2200

[3] Song Xu. CAGD on the C-Bézier curves and surfaces malleability research. Nanjing University of Aeronautics and Astronautics master's degree thesis, 2004:22-35

[4] Qinmin Yang, Guozhao Wang. Inflection point sand singularities on Ccurve[J]. 21:207-213

[5] He Lingna, Wang Guozhao Five C- curve segments [J]. Journal of Zhejiang University (Natural Science), 31(2), 2004:156-189

[6] LüY, G X. Uniform hyperbolic polynomial B-Spline curves[J]. CAGD, 2002, 19:379-393

[7] Forrest A. R. Interactive interpolation and approximation by Béziercurve [J]. ThecomputerJournal, 1972, 15(1):71-79

[8] Park Y, Choi U J. The error analysis for degree reduction of Béziercurves [J]. A Journal of Computers and Mathematics with Application, 1994, 27(12):1-6

[9] HoschekJ. Approximation of spline curves [J]. CAGD, 1987, 4(1):59-66

[10] Watkins M, Worsey A. Degree reduction for Béziercurves [J]. CAD 1988, 20(7):398-405

[11] Ye Zheng lin, Wei Sheng min,, Feng Guo sheng. On properties of planar parametrictension [J]. Journal of North western Polytechnical University, 1995, 13(3):458-462

[12] Buchin Su. Hesheng Hu, Yuanlong Xin. Differential geometry [M] Beijing: Higher Education Press, 1990
[13] Jianming Zheng, Thomas W. Gaussian and mean curvatures fractional Bézierpatches [J]. CAGD20, 2003:297-301 